\title{
FEEDBACK STABILIZATION OF “HYBRID” BILINEAR SYSTEMS*
}

\author{
BY \\ M. SLEMROD ${ }^{1}$ (Rensselaer Polytechnic Institute) \\ AND \\ E. L. ROGERS ${ }^{1}$ (Siena College)
}

\begin{abstract}
This paper considers the problem of stabilizing a control system governed by a combination of partial and ordinary differential equations. The partial differential equations govern the evolution of the system in the interior of some spatial domain, and the ordinary differential equations describe the evolution of the boundary data; the control enters through the boundary ordinary differential equations in a bilinear fashion. We provide sufficient conditions for feedback stabilization of such "hybrid" systems. Two examples to wave equations with dynamic boundary conditions are provided.
\end{abstract}

0. Introduction. In a recent paper [1] Burns and Cliff formulated a model of a "hybrid" system, i.e., a mixed system of partial and ordinary differential equations, in which the control enters only within the context of the ordinary differential equations. Interest in such systems can be motivated by problems in structures in which the control dynamics take place only in the boundary conditions of a distributed parameter system. In this paper we consider two problems (motivated by the example of Burns and Cliff) where the control enters the boundary conditions in a bilinear fashion. Our goal is to synthesize feedback controls that will stabilize the originally critically stable systems, i.e., we wish to find feedback controls so that the states of the feedback systems approach the zero state as $t \rightarrow \infty$.

The main tool of our analysis will be the "hyperbolic" stabilization theory of Ball and Slemrod [2, 3]. In fact, the theory of [2,3] may be readily applied to our problem.

The paper is divided into five parts. Sec. 1 recalls the results on feedback stabilization of $[2,3]$. Sec. 2 introduces the first hybrid system. Sec. 3 shows the feedback stabilizability of

\footnotetext{
${ }^{*}$ Received October 24, 1985 .

${ }^{1}$ Sponsored by the United States Army under Contract No. DAAG29-80-C-0041 and in part by the Air Force Office of Scientific Research, Air Force Systems Command, USAF, under Contract/Grant No. AFOSR-81-0172. The United States Government is authorized to reproduce and distribute reprints for Government purposes not withstanding any copyright hereon.
} 
the first hybrid system. Similarly, Sec. 4 discusses the second hybrid system and Sec. 5 proves feedback stabilizability for it.

1. Feedback stabilization. Consider the initial value problem

$$
\dot{u}(t)=\mathscr{A} u(t)+p(t) \mathscr{B} u(t), \quad u(0)=u_{0} \in X
$$

where $\mathscr{A}$ is the infinitesimal generator of a $C_{0}$ semigroup of contractions $e^{\mathscr{A} t}$ on a real Hilbert space $X, \mathscr{B}$ is a bounded linear operator $X \rightarrow X$, and $p(t)$ is a real-valued control. $X$ is endowed with inner product $\langle\cdot, \cdot\rangle_{X}$.

DEFINITION. System $(\mathscr{P})$ is stabilized (weakly stabilizable) if there exists a continuous feedback control $p: X \rightarrow \mathbf{R}$ such that $(\mathscr{P})$ with $p(t)=p(u(t))$ satisfies the following properties. $(\mathscr{P})$.

(i) For each $u_{0}$ there exists a unique weak solution of $u\left(t ; u_{0}\right)$ defined for all $t \in \mathbf{R}$ of

(ii) $\{0\}$ is a stable equilibrium of $(\mathscr{P})$.

(iii) $u\left(t, u_{0}\right) \rightarrow 0\left(u\left(t, u_{0}\right) \rightarrow 0\right.$ weakly) as $t \rightarrow \infty$ in $X$ for all $u_{0} \in X$.

The natural approach to the stabilization problem is to differentiate $\|u(t)\|_{X}^{2}=$ $\langle u(t), u(t)\rangle_{X}$ along trajectories of $(\mathscr{P})$. In this manner we obtain

$$
(d / d t)\|u(t)\|_{X}^{2}=2\langle\mathscr{A} u(t), u(t)\rangle_{X}+2 p(t)\langle u(t), \mathscr{B} u(t)\rangle_{X},
$$

at least formally. An obvious choice of feedback control (though not the only one) is

$$
p(u)=-\langle u, \mathscr{B} u\rangle_{X}
$$

since this control yields the "dissipative energy inequality"

$$
(d / d t)\|u(t)\|_{X}^{2} \leqslant-2\langle u(t), \mathscr{B} u(t)\rangle_{X}^{2} .
$$

(Note that $e^{\mathscr{A} t}$ a contraction means $\langle\mathscr{A} u(t), u(t)\rangle_{X} \leqslant 0$ for $u(t) \in D(\mathscr{A})$ ). So formally this choice of control $p(u)$ yields a feedback system of that form

$$
\dot{u}(t)=\mathscr{A} u(t)-\langle u(t), \mathscr{B} u(t)\rangle_{X} \mathscr{B} u(t) .
$$

For the purposes of this paper the following theorem of Ball and Slemrod [3] will be needed.

THEOREM 1. If $\mathscr{B}: X \rightarrow X$ is compact and

$$
\left\langle e^{\mathscr{A}} \psi, \mathscr{B} e^{\mathscr{A}} \psi\right\rangle_{X}=0 \quad \text { for all } t \in \mathbf{R}^{+} \Rightarrow \psi=0
$$

then $(\mathscr{P})$ is weakly stabilizable.

In some applications it will be convenient to work with second-order "hyperbolic" systems of the form

$$
\ddot{y}(t)+A y(t)+p(t) B y(t)=0, \quad y(0)=y_{0} \in H_{A}, \quad \dot{y}(0)=y_{1} \in H .
$$

Here $H$ is a real Hilbert space with inner product $\langle\cdot, \cdot\rangle_{H}$ and norm $\|\cdot\|_{H}^{2}=\langle\cdot, \cdot\rangle_{H}$. $A$ is a densely defined positive selfadjoint operator on $H$ such that $A^{-1}$ is everywhere 
defined and compact. We suppose the eigenvalues $\lambda_{n}^{2}$ of $A, n=1,2, \ldots, 0<\lambda_{1}<\lambda_{2}<$ $\cdots$, are simple. We denote the corresponding sequence of eigenfunctions by $\left\{\phi_{n}\right\}$. Let $H_{A}=D\left(A^{1 / 2}\right) . H_{A}$ form a Hilbert space under the inner product

$$
\left\langle y, y^{*}\right\rangle_{A}=\left\langle A^{1 / 2} y, A^{1 / 2} y^{*}\right\rangle .
$$

Denote $\|\cdot\|_{A}$ as the norm of $H_{A}$. We assume $B$ is a bounded linear map: $H_{A} \rightarrow H$. Again the function $p(t)$ is a scalar real-valued control.

If we set

$$
\begin{gathered}
u(t)=\left(\begin{array}{c}
y(t) \\
\dot{y}(t)
\end{array}\right), \quad \mathscr{A}=\left(\begin{array}{cc}
0 & I \\
-A & 0
\end{array}\right), \\
\mathscr{B}=\left(\begin{array}{cc}
0 & 0 \\
-B & 0
\end{array}\right), \quad D(\mathscr{A})=D(A) \times H_{A}, \\
X=H_{A} \times H \quad \text { with }\left\langle(y, \dot{y}),\left(y^{*}, \dot{y}^{*}\right)\right\rangle_{X}=\left\langle y, y^{*}\right\rangle_{H_{A}}+\left\langle\dot{y}, \dot{y}^{*}\right\rangle_{H},
\end{gathered}
$$

we see $\mathscr{A}, \mathscr{B}$ are required for problem $(\mathscr{P})$ above. We can now state the theorem of Ball and Slemrod given above in a second-order context (see [2] for a generalization).

TheOREM 2. Suppose $A$ and $B$ are as above. Assume $B: H_{A} \rightarrow H$ is compact and

$$
\begin{gathered}
\left\langle B \phi_{k}, \phi_{k}\right\rangle_{H} \neq 0 \text { for } k=1,2, \ldots, \\
\lambda_{m} \pm \lambda_{n} \neq 2 \lambda_{k} \quad \text { unless } m=n=k \text { and the }+ \text { sign is taken, } \\
\text { or both }\left\langle B \phi_{m}, \phi_{n}\right\rangle_{H} \text { and }\left\langle B \phi_{n}, \phi_{m}\right\rangle_{H} \text { are zero. }
\end{gathered}
$$

Then the feedback system

$$
\ddot{y}(t)+A y(t)+\langle B y(t), \dot{y}(t)\rangle_{H} \mathscr{B} y(t)=0
$$

and the initial data $y(0)=y_{0} \in H_{A}, \dot{y}(0)=y_{1} \in H$ possess a unique globally defined weak solution $(y, \dot{y}) \in C\left((0, \infty) ; H_{A} \times H\right)$ and $(y(t), \dot{y}(t)) \rightarrow(0,0)$ weakly in $X=H_{A}$ $\times H$ as $t \rightarrow \infty$ and $\left(\mathscr{P}^{\prime}\right)$ is weakly stabilizable.

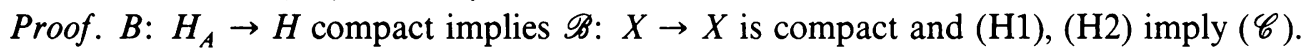
See [2] for details.

2. Hybrid system 1. Motivated by the example in [1] we consider the string-mass system shown in Fig. 1. The string has length 1, constant linear density $\sigma$, and is under constant tension $\tau$. The purpose of the device at the right end is to maintain the tension; however, it is idealized so as to provide no impedance to the vertical motion at the end.

The vertical motion of the string is assumed to satisfy the linear wave equation

$$
z_{t t}(t, x)=\alpha^{2} z_{x x}(t, x), \quad 0<x<1,
$$

where $\alpha^{2}=\tau / \sigma>0$. The motion of the right end of the string is governed by the balance of linear momentum. The relevant forces here are acceleration of the point mass $m$, the tensile form on the string $\tau z_{x}(t, 1)$, and external forces. We assume we can impose an external force at the right end in the bilinear form $p(t) z(t, 1)$. In the absence of other external forces, the equation of balance of linear momentum of the mass $m$ is

$$
m z_{t t}(t, 1)=-p(t) z(t, 1)-\tau z_{x}(t, 1) .
$$




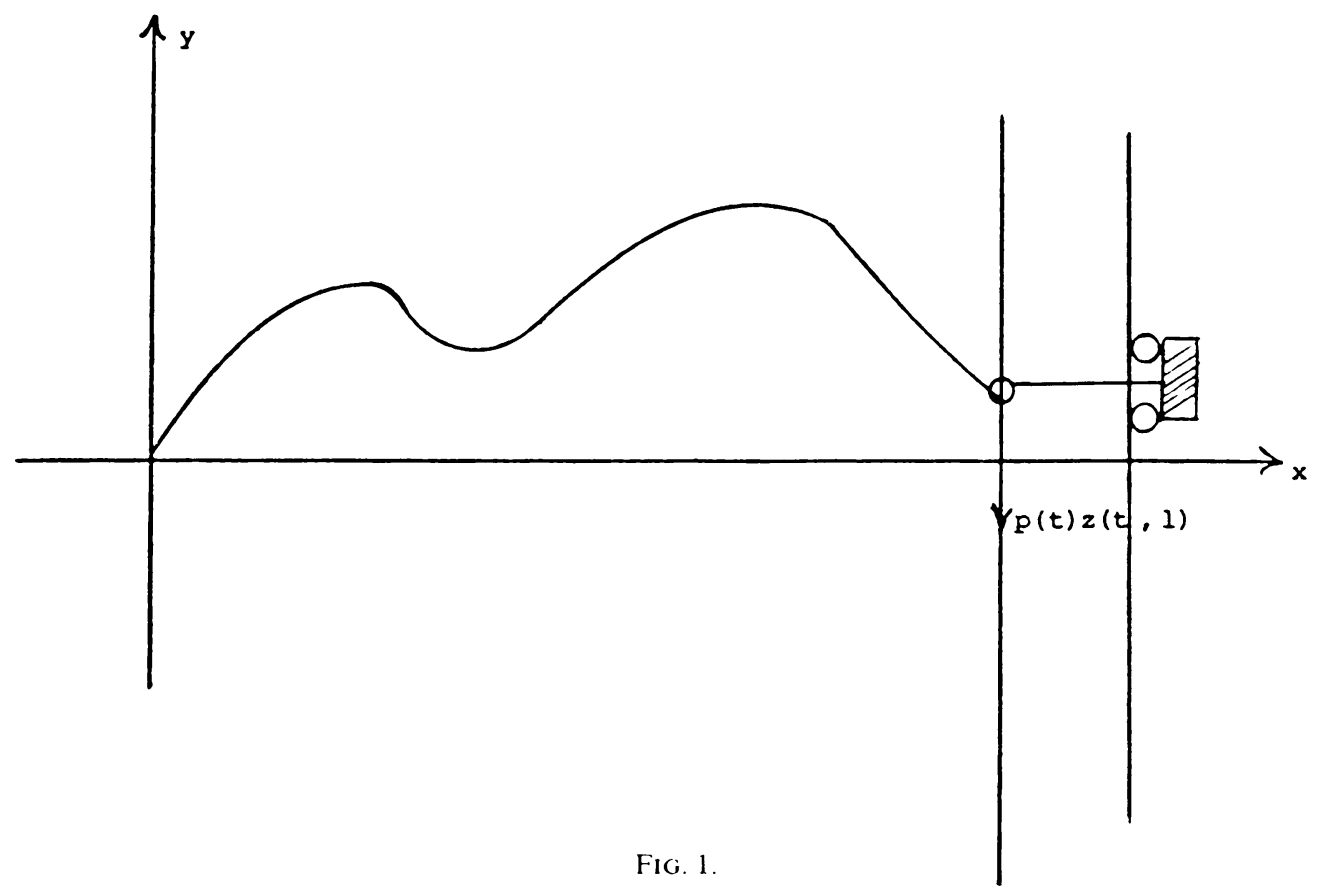

The left end is assumed fixed at $x=0$, so that

$$
z(t, 0)=0 \text {. }
$$

The initial conditions are

$$
z(0, x)=f(x), \quad z_{t}(0, x)=g(x), \quad 0<x<1 .
$$

3. Abstract formulation of hybrid system and feedback stabilization. For the hybrid system (2.1)-(2.4), set

$$
y(t)=\left(\begin{array}{c}
z(t, x) \\
z_{1}(t)
\end{array}\right) \text { where } z_{1}(t)=z(t, 1)
$$

Also define the operators

$$
A=\left(\begin{array}{cc}
-\alpha^{2} \frac{d^{2}}{d x^{2}} & 0 \\
\left.\frac{\tau}{m} \frac{d}{d x}\right|_{x=1} & 0
\end{array}\right), \quad B=\left(\begin{array}{cc}
0 & 0 \\
0 & 1 / m
\end{array}\right),
$$

where $D(A)=\left\{\left(z, z_{1}\right) \in H^{2}(0,1) \times \mathbf{R} ; z(0)=0, z(1)=z_{1}\right\}$. Set $H=L^{2}(0,1) \times \mathbf{R}$, where $H$ is a Hilbert space endowed with the inner product

$$
\left\langle(v, w),\left(v^{*}, w^{*}\right)\right\rangle_{H}=\frac{1}{\alpha^{2}} \int_{0}^{1} v(x) v^{*}(x) d x+\frac{m}{\tau} w w^{*} .
$$

Clearly $D(A)$ is dense in $H$. Of course we see that now (2.1)-(2.4) has the form (1.1). 
Lemma 1. $A$ is a positive definite, selfadjoint operator on $H$, with bounded inverse $A^{-1}$.

Proof. We first prove $A$ is symmetric, i.e., $\left\langle A(v, w),\left(v^{*}, w^{*}\right)\right\rangle_{H}=\left\langle(v, w), A\left(v^{*}, w^{*}\right)\right\rangle_{H}$ for all $(v, w),\left(v^{*}, w^{*}\right) \in D(A)$ where $D(A) \subset D\left(A^{*}\right)$. To see this, simply compute

$$
\begin{aligned}
\left\langle A(v, w),\left(v^{*}, w^{*}\right)\right\rangle_{H} & =\int_{0}^{1} v^{\prime}(x) v^{*}(x) d x \\
& =\left\langle(v, w), A\left(v^{*}, w^{*}\right)\right\rangle_{H} .
\end{aligned}
$$

Furthermore, since for $(v, w) \in D(A)$ we have $v(x)=\int_{0}^{x} v^{\prime}(x) d x \leqslant\left(\int_{0}^{1} v^{\prime}(x)^{2} d x\right)^{1 / 2}$ and hence the following inequality holds:

$$
\sup _{0 \leqslant x \leqslant 1}|v(x)|^{2}+\int_{0}^{1} v(x)^{2} d x \leqslant \text { const. } \int_{0}^{1} v^{\prime}(x)^{2} d x .
$$

Thus from (3.1), we have

$$
\langle A(v, w),(v, w)\rangle_{H} \geqslant \text { const. }\|(v, w)\|_{H}^{2}, \quad \text { const. }>0,
$$

i.e., the operator $A$ is accretive. By the Schwarz inequality,

$$
\|A(v, w)\|_{H}\|(v, w)\|_{H} \geqslant \text { const. }\|(v, w)\|_{H}^{2},
$$

and hence

$$
\|A(v, w)\|_{H} \geqslant \text { const. }\|(v, w)\|_{H} .
$$

Thus $A$ possesses a bounded inverse on $H$. Hence $R(A)=H$ and a well-known result (e.g., see Yosida [4; p. 199]) shows $D\left(A^{*}\right) \subset D(A)$ and $A$ is selfadjoint. Inequality (3.3) shows that $A$ is positive definite.

LemMa 2. $A^{-1}: H \rightarrow H$ is compact.

Proof. Let $\left\{\left(v_{n}, w_{n}\right)\right\}$ be a bounded sequence in $H$ and set $A^{-1}\left(v_{n}, w_{n}\right)=\left(z_{n}, z_{1 n}\right)$, $n=1,2, \ldots$. Since $A^{-1}$ is bounded, $\left\|\left(z_{n}, z_{1 n}\right)\right\|_{H} \leqslant$ const. for all $n$. Also, since

$$
-\alpha^{2} \frac{d^{2}}{d x^{2}} z_{n}(x)=v_{n}(x)
$$

we have

$$
\frac{\tau}{m} \frac{d z_{n}(1)}{d x}=w_{n}
$$

Multiplication of (3.5a) by $z_{n}$ and integration by parts yield

$$
\begin{aligned}
\alpha^{2} \int_{0}^{1} z_{n}^{\prime}(x)^{2} d x & =\alpha^{2} z_{n}(1) z_{n}^{\prime}(1)+\int_{0}^{1} z_{n}(x) v_{n}(x) d x \\
& =\alpha^{2} \frac{m}{\tau} z_{n}(1) w_{n}+\int_{0}^{1} z_{n}(x) v_{n}(x) d x \\
& =\alpha^{2}\left\langle\left(z_{n}, z_{1 n}\right),\left(v_{n}, w_{n}\right)\right\rangle \\
& \leqslant \alpha^{2}\left\|\left(z_{n}, z_{1 n}\right)\right\|_{H}\left\|\left(v_{n}, w_{n}\right)\right\|_{H} \leqslant \text { const. }
\end{aligned}
$$

Since $\int_{0}^{1} z_{n}^{\prime 2}(x) d x \geqslant \int_{0}^{2} z^{2}(x) d x$ by (3.2), we know $\left\{z_{n}\right\}$ lies in a bounded set of $H^{1}(0,1)$. Since the injection of $H^{1}(0,1)$ into $L^{2}(0,1)$ is compact, $\left\{z_{n}\right\}$ possesses a convergent subsequence in $L^{1}(0,1)$. Since $\left\{z_{1 n}\right\}$ is a bounded sequence in $\mathbf{R}$, it certainly contains a 
bounded subsequence. Hence $A^{-1}$ maps bounded sets of $H$ into precompact sets of $H$ and hence is compact.

Lemma 3. The eigenvalues of $A$ are given by a sequence $0<\lambda_{1}^{2}<\lambda_{2}^{2}<\cdots$, where $\left\{\lambda_{n}\right\}$ are the increasing positive roots of

$$
\tau / m \alpha \lambda=\tan (\lambda / \alpha)
$$

$\left\{\left(\sin \lambda_{n} x, \sin \lambda_{n}\right)\right\}$ are the associated eigenvectors.

Proof. If $\lambda^{2}$ is an eigenvalue of $A$, then

$$
-\alpha^{2} \frac{d^{2} z}{d x^{2}}=\lambda^{2} z, \quad 0<x<1, \quad \frac{\tau}{m} \frac{d z(1)}{d x}=\lambda^{2} z(1),
$$

and $z(0)=0$, where $(z(x), z(1))$ is the associated eigenvector. Thus $z(x)=\sin (\lambda / \alpha) x$ and $\tau / m \alpha \lambda=\tan (\lambda / \alpha)$.

LEMMA 4. $B$ is a bounded linear selfadjoint operator: $H \rightarrow H$.

Proof. $\|B(v, w)\|_{H}=\|(0, w / m)\|_{H} \leqslant$ const. $\|(v, w)\|_{H}$, so $B$ is bounded. Also $\langle B(v, w)$, $\left.\left(v^{*}, w^{*}\right)\right\rangle_{H}=w w^{*} / \tau=\left\langle(v, w), B\left(v^{*}, w^{*}\right)\right\rangle_{H}$, so $B$ is symmetric and bounded, hence selfadjoint.

LEMMA 5.

$$
\left\langle B\left(\sin \lambda_{n} x, \sin \lambda_{n}\right),\left(\sin \lambda_{n} x, \sin \lambda_{n}\right)\right\rangle_{H} \neq 0 \text { for } n=1,2, \ldots
$$

Proof.

$$
\left\langle B\left(\sin \lambda_{n} x, \sin \lambda_{n}\right),\left(\sin \lambda_{n} x, \sin \lambda_{n}\right)\right\rangle_{H}=\left(\sin ^{2} \lambda_{n}\right) / \tau \neq 0 .
$$

Lemma 6. For the eigenvalues of $A$, we know that $\lambda_{m} \pm \lambda_{n} \neq 2 \lambda_{k}$ unless $m=n=k$ and the + sign is taken.

Proof. Assume $\lambda_{m} \pm \lambda_{n}=2 \lambda_{k}$. Divide by $\alpha$ and take tan of both sides. We then have

$$
\tan \left(\lambda_{m} / \alpha \pm \lambda_{n} / \alpha\right)=\tan \left(2 \lambda_{k} / \alpha\right)
$$

which in turn implies, by the usual trigonometric identities,

$$
\frac{\tan \left(\lambda_{m} / \alpha\right) \pm \tan \left(\lambda_{n} / \alpha\right)}{1 \mp \tan \left(\lambda_{m} / \alpha\right) \tan \left(\lambda_{n} / \alpha\right)}=\frac{2 \tan \left(\lambda_{k} / \alpha\right)}{1-\tan ^{2}\left(\lambda_{k} / \alpha\right)}
$$

We now use the definition of $\lambda_{n}$ (Lemma 3$)$ to assert

$$
\frac{(\tau / m \alpha)\left(\lambda_{m}^{-1} \pm \lambda_{n}^{-1}\right)}{1 \mp(\tau / m \alpha)^{2} \lambda_{m}^{-1} \lambda_{n}^{-1}}=\frac{2(\tau / m \alpha) \lambda_{k}^{-1}}{1-(\tau / m \alpha)^{2} \lambda_{k}^{-2}}
$$

or

$$
\frac{\left(\lambda_{n} \pm \lambda_{m}\right)}{\lambda_{m} \lambda_{n} \mp(\tau / m \alpha)^{2}}=\frac{2 \lambda_{k}}{\lambda_{k}^{2}-(\tau / m \alpha)^{2}} .
$$

We now have two cases to consider.

Case 1. $\lambda_{m}+\lambda_{n}=2 \lambda_{k}$. In this case (3.7) implies $\lambda_{m} \lambda_{n}=\lambda_{k}^{2}$. But since $\lambda_{m}^{2}+\lambda_{n}^{2}+$ $2 \lambda_{m} \lambda_{n}=4 \lambda_{k}^{2}$, we see $\left(\lambda_{m}-\lambda_{n}\right)^{2}=0$ and $\lambda_{m}=\lambda_{n}=\lambda_{k}$.

Case 2. $\lambda_{m}-\lambda_{n}=2 \lambda_{k}$. In this case $\lambda_{n}-\lambda_{m}=-2 \lambda_{k}$ and (3.4) implies

$$
\lambda_{m} \lambda_{n}+(\tau / m \alpha)^{2}=-\lambda_{k}^{2}+(\tau / m \alpha)^{2}
$$

or $\lambda_{m} \lambda_{n}=-\lambda_{k}^{2}$, which contradicts the positivity of $\left\{\lambda_{n}\right\}$. This completes the proof. 
THEOREM 3. The feedback system (2.1), (2.2) with

$$
p(t)=z_{t}(t, 1) z(t, 1) / m
$$

possesses a unique, globally defined weak solution for initial data $(f, f(1)) \in H_{A},(g, g(1))$ $\in H,\left(\left(y, y_{1}\right),\left(\dot{y}, \dot{y}_{1}\right)\right) \rightarrow((0,0),(0,0))$ weakly in $H_{A} \times H$ as $t \rightarrow \infty$, and (2.1), (2.2) is weakly stabilizable in $H_{A} \times H$.

Proof. Lemmas 1-6 show that the hypotheses of Theorem 2 are satisfied.

4. The hybrid system 2 . Suppose $G$ is a domain in $\mathbf{R}^{3}$ filled with a compressible fluid which is at rest except for acoustic wave motion. If $\phi(x, y, z, t)$ is the velocity potential, so that $-\nabla \phi$ is the particle velocity, then linearized theory says that $\phi$ satisfies the wave equation

$$
\phi_{t t}=c^{2} \Delta \phi \quad \text { in } G,
$$

where $c$ is the speed of sound in the medium, $\phi_{t}$ is the pressure distribution ( $\doteq$ $w(x, y, z, t))$. Now suppose that the boundary of $G, \partial G$, possesses a nonrigid section $\Gamma_{1}$ which is subject to small oscillations. We assume that each point on $\Gamma_{1}$ reacts to the excess pressure of the acoustic wave like a harmonic oscillator. We assume also that different parts of the boundary do not influence each other, so that $\Gamma_{1}$ is locally reacting. Then the normal displacement $\delta$ of the boundary $\Gamma_{1}$ into $G$ satisfies an equation of the form

$$
m \delta_{t t}+k \delta=-\rho \phi_{t}+f \text { on } \Gamma_{1},
$$

where $\rho$ is the (assumed constant in linear theory) density of the fluid, $m, k$ are positive constants, and $f$ is an applied external force on $\Gamma_{1}$. If we assume the boundary section $\Gamma_{1}$ is impenetrable, we obtain a third equation from the continuity of velocity at the boundary,

$$
\delta_{t}=\partial \phi / \partial n \text { on } \Gamma_{1},
$$

where $\partial \phi / \partial n$ is the outward normal velocity. (This model has been given in [5, p. 263]; a mathematical analysis is found in [6], and a discussion of linear control synthesis appears in [7].)

If we assume $G$ is bounded and $\Gamma_{1} \cup \Gamma_{2} \cup \Gamma_{3}=\partial G$, where $\Gamma_{2}$ is a free surface, then the pressure on $\Gamma_{2}$ must take on the ambient atmospheric pressure distribution. If we normalize this boundary pressure distribution to zero, we find

$$
\phi_{t}=0 \text { on } \Gamma_{2} \text {. }
$$

$\Gamma_{3}$ is a rigid boundary so

$$
\frac{\partial \phi}{\partial n}=0 \text { on } \Gamma_{3} .
$$

In this paper we consider the case where $G$ is a right circular cylinder with free surface at the top and reacting boundary at the bottom. This is shown in Fig. 2.

For simplicity we set $c=\rho=m=k=1$. Also we seek a solution $\phi=\phi(x, t)$ of (4.1)-(4.5). In this case, (4.5) is automatically satisfied. Differentiation of (4.1)-(4.4) with respect to $t$ yields the system

$$
\begin{gathered}
w_{t t}=w_{x x}, \quad 0<x<1, \\
\theta_{t t}+\theta=-w_{t}(t, 1)+v(t) w(t, 1), \quad x=1,
\end{gathered}
$$




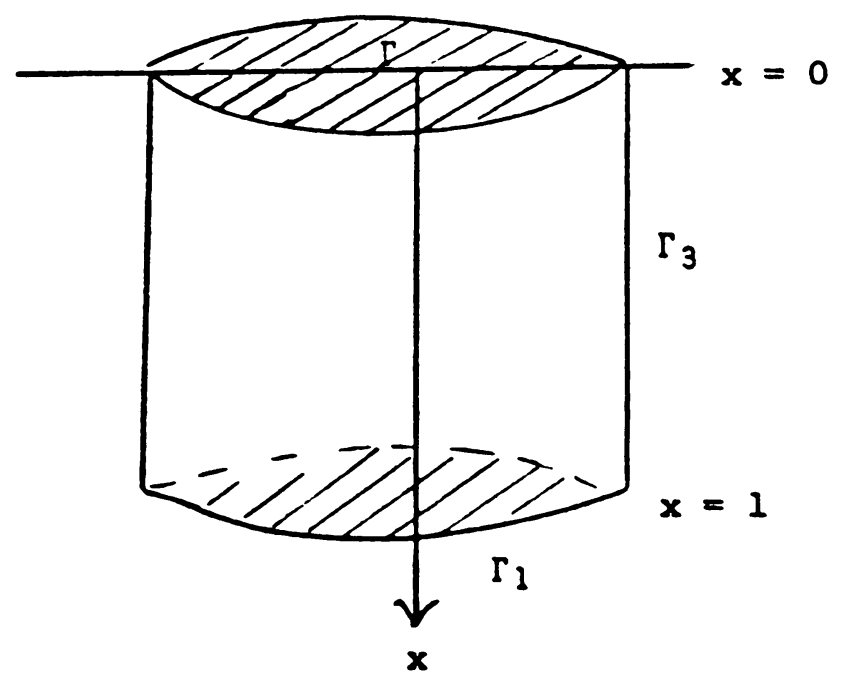

FIG. 2.

$$
\begin{gathered}
\theta_{t}=w_{x}, \quad x=1, \\
w=0, \quad x=0,
\end{gathered}
$$

where $\theta \equiv \delta_{t}$, and we have synthesized the external driving force $f^{\prime}(t)=v(t) w(t, 1)$, i.e., we are looking for a feedback which then multiplies the observed pressure at $x=1$. Of course, we must also specify initial conditions

$$
w(0, x)=w_{0}(x), \quad w_{t}(0, x)=w_{1}(x), \quad \theta(0)=\theta_{0}, \quad \theta_{t}(0)=\theta_{1}, \quad 0<x<1 .
$$

5. Abstract formulation of hybrid system 2 and feedback stabilization. For the hybrid system $\left(4.1^{\prime}\right)-\left(4.4^{\prime}\right)$, set

$$
\begin{gathered}
u=\left(\begin{array}{c}
w \\
w_{t} \\
\theta \\
\theta_{t}
\end{array}\right), \quad \mathscr{A} u=\left(\begin{array}{c}
w_{t} \\
w_{x x} \\
\theta_{t} \\
-\theta-w_{t}(\cdot, 1)
\end{array}\right), \quad \mathscr{B} u=\left(\begin{array}{c}
0 \\
0 \\
0 \\
w(\cdot, 1)
\end{array}\right), \\
X=\left\{\left(w, w_{t}, \theta, \theta_{t}\right) ; w \in H^{1}(0,1), w_{t} \in L_{2}(0,1), \theta \in \mathbf{R}, \theta_{t} \in \mathbf{R}, w=0 \text { at } x=0\right\}
\end{gathered}
$$

endowed with inner product

$$
\begin{gathered}
\left\langle u, u^{*}\right\rangle_{X}=\int_{0}^{1}\left(w_{x} w_{x}^{*}+w_{t} w_{t}^{*}\right) d x+\theta \theta^{*}+\theta_{t} \theta_{t}^{*}, \\
D(\mathscr{A})=\left\{\left(w, w_{t}, \theta, \theta_{t}\right) \in X ; w \in H^{2}(0,1), w_{t} \in H^{1}(0,1),\right. \\
\left.w_{t}=0 \text { at } x=0, \theta_{t}=w_{x} \text { at } x=1\right\} .
\end{gathered}
$$

Since for $w \in H^{1}(0,1)$, the Sobolev lemma ([4, p. 174]) says $w$ can be viewed as an element of $C(0,1)$ after possible modification on a set of measure zero, we shall take such $w$ as continuous. So for $w \in H^{1}(0,1), w=0, w$ is identified as a continuous function that satisfies

$$
w(x)=\int_{0}^{x} w^{\prime}(x) d x
$$


in the Lebesgue sense. Hence the boundary value $w(1)$ is simply

$$
w(1)=\int_{0}^{1} w^{\prime}(x) d x
$$

It is in this sense that the boundary conditions in $X$ and $D(\mathscr{A})$ are to be understood.

We note for $u \in D(\mathscr{A}),\langle\mathscr{A} u, u\rangle_{X}=0$ so $\mathscr{A}$ is certainly dissipative. $\mathscr{A}$ is also densely defined and Range $(I-\mathscr{A})=X$ (by direct computation), so the Lumer-Phillips theorem [4] implies that $\mathscr{A}$ is the infinitesimal generator of a $C_{0}$ semigroup of contractions $e^{\mathscr{A} t}$ on $X$. Also, we note that if $\left\{u_{n}\right\}$ is a bounded sequence in $X,(5.1)$ shows $\left\{w_{n}(1)\right\}$ to be a bounded sequence in $\mathbf{R}$. Hence it possesses a convergent subsequence. Thus $\mathscr{B}: X \rightarrow X$ is compact.

All the hypotheses of Theorem 1 are satisfied modulo showing that the only solution of $\left\langle e^{\mathscr{A} t} \psi, \mathscr{B} e^{\mathscr{A} t} \psi\right\rangle=0$ for all $t \in \mathbf{R}^{+}$is $\psi \equiv 0$. In this problem

$$
\left\langle e^{\mathscr{A} t} \psi, \mathscr{B} e^{\mathscr{A} t} \psi\right\rangle=\tilde{\theta}_{t} \tilde{w}(1, t),
$$

where $\tilde{w}, \tilde{\theta}$ are solutions to the uncontrolled $(p \equiv 0)$ system $\left(4.1^{\prime}\right)-\left(4.4^{\prime}\right)$. Separation of variables shows

$$
\begin{aligned}
& \tilde{w}(x, t)=\sum_{k=1}^{\infty}\left(A_{k} e^{i \sigma_{k} t}+\overline{A_{k}} e^{-i \sigma_{k} t}\right) \sin \sigma_{k} x, \\
& \tilde{\theta}_{t}(t)=\sum_{k=1}^{\infty}\left(A_{k} e^{i \theta_{k} t}+\bar{A}_{k} e^{-i \sigma_{k} t}\right) \sigma_{k} \cos \sigma_{k},
\end{aligned}
$$

where $\sigma_{k}$ are the positive roots of

$$
\tan \sigma=1 / \sigma-\sigma .
$$

Here - denotes the complex conjugate. If we substitute (5.2), (5.3) into the equation $\tilde{\theta}_{t} \tilde{w}(1, t)=0$ for all $t \in \mathbf{R}^{+}$, we obtain

$$
\begin{aligned}
2 \sum_{k=1}^{\infty}\left|A_{k}\right|^{2} \sigma_{k} \sin \sigma_{k} \cos \sigma_{k}+\sum_{k=1}^{\infty}\left(A_{k}^{2} e^{i 2 \sigma_{k} t}+\overline{A_{k}^{2}} e^{-i 2 \sigma_{k} t}\right) \sigma_{k} \sin \sigma_{k} \cos \sigma_{k} \\
\quad+\sum_{\substack{n, k=1 \\
n \neq k}}^{\infty}\left(A_{k} A_{n} e^{i\left(\sigma_{n}+\sigma_{k}\right) t}+\overline{A_{n}} \overline{A_{k}} e^{i\left(\sigma_{n}-\sigma_{k}\right) t}\right. \\
+A_{n} \overline{A_{k}} e^{i\left(\sigma_{n}-\sigma_{k}\right) t}+\overline{A_{n}} \overline{A_{k}} e^{\left.i\left(\sigma_{k}-\sigma_{n}\right) t\right)} \sigma_{n} \sin \sigma_{k} \cos \sigma_{n}=0 \quad \text { for all } t \in \mathbf{R}^{+} .
\end{aligned}
$$

The terms on the left side of (5.5) form an almost periodic function in $t$. If none of the frequencies appearing in the second sum appear in the first and third sums, the uniqueness theorem for almost periodic functions [8] will imply $A_{k}=0, k=1,2, \ldots$ This in turn will imply $\tilde{w}(x, t)=0, \tilde{\theta}_{t}(t)=0$ which with $\left(4.2^{\prime}\right)$ shows $\psi \equiv 0$ to be the only solution of $\left\langle e^{\mathscr{A} t} \psi, \mathscr{B} e^{\mathscr{A} t} \psi\right\rangle=0$ for all $t \in \mathbf{R}^{+}$. So we now prove the following lemma.

LEMmA 7. Let $\sigma_{n}$ be the positive roots of (5.4), $0<\sigma_{1}<\sigma_{2}<\sigma_{3} \cdots$. Then $\sigma_{r} \pm \sigma_{s}=2 \sigma_{k}$ holds only when $r=s=k$ and + sign is taken. 
Proof. For later use we record the first three positive roots of (5.4):

$$
\sigma_{1}=0.67625 \cdots, \quad \sigma_{2}=2.11708 \cdots, \quad \sigma_{3}=4.92125 \cdots .
$$

Case 1. Assume $\sigma_{r}+\sigma_{s}=2 \sigma_{k}$. Then taking tan of both sides and using (5.4) we find

$$
\frac{1-\sigma_{r} \sigma_{s}}{4 \sigma_{k}^{2}-3 \sigma_{r} \sigma_{s}-\left(1-\sigma_{r} \sigma_{s}\right)^{2}}=\frac{1-\sigma_{k}^{2}}{\sigma_{k}^{2}-\left(1-\sigma_{k}^{2}\right)^{2}} .
$$

Solving for $\sigma_{r} \sigma_{s}$, we see

$$
\sigma_{r} \sigma_{s}=\frac{3 \sigma_{k}^{2}-2}{1-\sigma_{k}^{2}}
$$

(Note $\sigma_{r} \sigma_{s} \neq 1, \sigma_{k}^{2} \neq 1$, so our manipulations are valid.) If $\sigma_{k}^{2}<2 / 3$ or $\sigma_{k}^{2}>1$, we see $\sigma_{r} \sigma_{s}<0$, which violates positivity of the roots. Since $\sigma_{k}^{2} \notin\left(\frac{2}{3}, 1\right)$ for all $k$, we see that $\sigma_{r}+\sigma_{s}=2 \sigma_{k}$ holds only when $\sigma_{r}=\sigma_{s}=\sigma_{k}$, i.e., $r=s=k$.

Case 2. Assume $\sigma_{r}-\sigma_{s}=2 \sigma_{k}$ and proceed as in Case 1. We find this time that

$$
\sigma_{k}^{2}=1+1 / \sigma_{r} \sigma_{s}-3 \text {. }
$$

If $r, s \geqslant 2, r \neq s$, graphical analysis of (5.4) shows $\sigma_{r}-\sigma_{s} \geqslant \sigma_{3}-\sigma_{2}=2.80417 \ldots$ So if $2 \sigma_{k}=\sigma_{r}-\sigma_{s}$, we must have $\sigma_{k}^{2}>1.965 \cdots$. Also we know that for $r, s \geqslant 2, \sigma_{r} \sigma_{s}>3$. Hence from (5.7), we see that if $\sigma_{r}-\sigma_{s}=2 \sigma_{k}$ for $r, s \geqslant 2, r \neq s$, then

$$
1.965 \cdots<1+1 / \sigma_{r} \sigma_{s}-3
$$

for $3<\sigma_{r} \sigma_{s}<4.035 \cdots$. No pair $\sigma_{r}, \sigma_{s}$ satisfies this relation.

On the other hand, for $r$ or $s<2$ we can only have $s=1, r>1$. We consider the set $\left\{\sigma_{r}\right\}$, so that

$$
1+\frac{1}{\sigma_{1} \sigma_{r}-3} \leqslant \sigma_{2}^{2}=4.48203 \cdots .
$$

This set is the set of $\left\{\sigma_{r}\right\}$, so that $\sigma_{1} \sigma_{r}>3.287186 \cdots$ or $\sigma_{r}>4.8609 \cdots$, i.e., $r \geqslant 3$. So for $r \geqslant 3$, the only way (5.7) can hold with $s=1$ is when $k=1$, i.e., $\sigma_{r}-\sigma_{1}=2 \sigma_{1}$ or $\sigma_{r}=3 \sigma_{1}$. But there is no such $\sigma_{r}$. Finally if $r=2$, we must have $\sigma_{2}-\sigma_{1}=2 \sigma_{k}$, i.e., $\sigma_{k}=.720415$, which also cannot hold. The lemma is proven.

We can now conclude from the discussion preceding Lemma 7 that the following theorem is true.

THEOREM 4. The feedback system $\left(4.1^{\prime}\right)-\left(4.4^{\prime}\right)$ with

$$
v(t)=-\theta_{t}(t) w(1, t)
$$

possesses a unique globally defined weak solution for initial data, $\left(w_{0}, w_{1}, \theta_{0}, \theta_{1}\right) \in X$ and $\left(w, w_{t}, \theta, \theta_{t}\right) \rightarrow(0,0,0,0)$ weakly in $X$ as $t \rightarrow \infty$ and $\left(4.1^{\prime}\right)-\left(4.4^{\prime}\right)$ is weakly stabilizable.

\section{REFERENCES}

[1] J. A. Burns and E. M. Cliff, On control and identification of hybrid systems, AIAA Symposium on Dynamics and Control of Large Space Structures, Blacksburg, Virginia (June, 1981)

[2] J. M. Ball and M. Slemrod, Nonharmonic Fourier series and the stabilization of distributed semi-linear control systems, Comm. Pure Appl. Math. XXXII, 555-587 (1979) 
[3] J. M. Ball and M. Slemrod, Feedback stabilization of distributed semilinear controls, Appl. Math. Optim. 5, 169-179 (1979)

[4] K. Yosida, Functional Analysis, Springer-Verlag, New York (1971)

[5] P. Morse and K. U. Ingard, Theoretical Acoustics, McGraw-Hill, New York (1968)

[6] J. T. Beale, Spectral properties of an acoustic boundary condition, Indiana Univ. Math. J. 25, 895-917 (1975)

[7] R. E. O'Brien, Energy decay in weakly locally reacting boundary value problems, Indiana Univ. Math. J. (in press)

[8] A. S. Besicovitch, Almost Periodic Functions, Dover, New York (1954) 